\title{
A deep mosaic of [O III]5007 $\AA$ CCD images of the environment of the LBV star P Cygni
}

\author{
P. Boumis ${ }^{1}$, J. Meaburn ${ }^{1,2}$, M. P. Redman ${ }^{3}$, and F. Mavromatakis ${ }^{4}$ \\ 1 Institute of Astronomy \& Astrophysics, National Observatory of Athens, I. Metaxa \& V. Paulou, P. Penteli, \\ 15236 Athens, Greece \\ e-mail: ptb@astro.noa.gr \\ 2 Jodrell Bank Observatory, University of Manchester, Macclesfield SK11 9DL, UK \\ 3 Dublin Institute for Advanced Studies, School of Cosmic Physics, 5 Merrion Square, Dublin 2, Republic of Ireland \\ 4 Technological Education Institute of Crete, General Department of Applied Science, PO Box 1939, GR-710 04 Heraklion, \\ Crete, Greece
}

Received 14 July 2006 / Accepted 1 August 2006

\section{ABSTRACT}

\begin{abstract}
A mosaic of six, deep, CCD images in the light of the [O III]5007 Å nebular emission line has been obtained with the 1.3-m Skinakas (Crete) telescope of the filamentary nebulosity surrounding P Cygni. The [O III] $5007 \AA$ Aine discriminates against confusing galactic HII regions along the same sight-lines and the new mosaic did not include the 4.8 mag. central star; a source of artifacts in the previous lower angular resolution observations. New giant "lobes" and "shells" are found to be clustered around P Cygni which must be the relics of historic eruptions between 2400 and up to $\approx 10^{5}$ yr ago.
\end{abstract}

Key words. stars: circumstellar matter - stars: variables: general - stars: individual: P Cygni

\section{Introduction}

The circumstellar environment of the proto-typical Luminous Blue Variable star (LBV - Conti 1984; Humphreys 1989; Davidson et al. 1989) P Cygni has been revealed at optical wavelengths in the work presented in a sequence of seven papers (Johnson et al. 1992; Barlow et al. 1994; Meaburn et al. 1996; O'Connor et al. 1998; Meaburn et al. 1999, 2000, 2004).

In these papers the discovery of a $22^{\prime \prime}$ diameter inner shell (IS) was found to be surrounded by a $1.6^{\prime}$ diameter, outer shell (OS). More controversially it was suggested that a large region of filamentary nebulosity which surrounds the star could have been ejected in the early stages of its evolution and not simply be an unrelated supernova remnant along the same sight-line. The latest kinematical and morphological evidence in support of this ejection proposition was presented in Meaburn et al. (2004) as a consequence of a deep, very wide-field, image in the light of the [O III]5007 $\AA$ nebular emission line, though still some reservations remained about the association with $\mathrm{P}$ Cygni. The "giant lobe" (GL) projecting to the east of P Cygni (O'Connor et al. 1998) was shown to have southern and eastern counterparts (here these will still be referred to as GLs). The wide-field CCD [O III]5007 $\AA$ image by Meaburn et al. (2004) was restricted in its sensitivity by the inclusion of the 4.8-mag star P Cygni in the same field; artifacts due to gross signal saturation and diffraction became unacceptable in places and areas of the final image had to be blanked out. Incidentally the [O III]5007 $\AA$ line was chosen for this imagery as this is dominant in the emission from the GL nebulosity; consequently considerable discrimination was achieved against the predominantly $\mathrm{H} \alpha$ and [N II]6584 $\AA$ emitting, diffuse, galactic HII regions in the broader field.
This saturation/artifact problem has since been overcome by obtaining a mosaic of deeper [O III]5007 $\AA$ CCD images at $\approx 5$ times higher angular resolution than previously covered fields adjacent to P Cygni but where none includes the star itself. New GL features that are revealed help to confirm - and see Sect. 3 - the association of all of the extensive, filamentary GL nebulosity with P Cygni.

\section{Observations and results}

A mosaic of 6 images was taken of the adjacent fields to P Cygni through an [O III]5007 A filter with the $1.3 \mathrm{~m}(f / 7.7)$ Ritchey-Cretien telescope at Skinakas Observatory in Crete, Greece on September 1-2, 5 and 9-10, 2005. The detector was a $1024 \times 1024$ (with $24 \times 24 \mu \mathrm{m}^{2}$ pixels) SITe CCD giving a field of view of $8 . .5 \times 88^{\prime \prime} .5$ and an image scale of 0.5 pixel $^{-1}$. One $2400 \mathrm{~s}$ exposure in the light of [O III]5007 $\AA$ and two $180 \mathrm{~s}$ exposures in the continuum were taken for each field to make sure that any cosmic ray hits will be identified and removed successfully (see Boumis et al. 2002 for the image processing details). The continuum filter was $230 \AA$ wide and centred on $5470 \AA$ and so did not transmit any strong emission line.

The image reduction was carried out using the IRAF package and their grey-scale representation generated using the STARLINK Kappa and Figaro packages. The astrometric solutions were calculated for each field using reference stars from the Hubble Space Telescope (HST) Guide Star Catalogue (Lasker et al. 1999). All coordinates quoted in this paper refer to epoch 2000.

Light and dark, negative, grey-scale representations of the same mosaic of [O III]5007 ̊̊ images are shown in Figs. 1 and 2, 


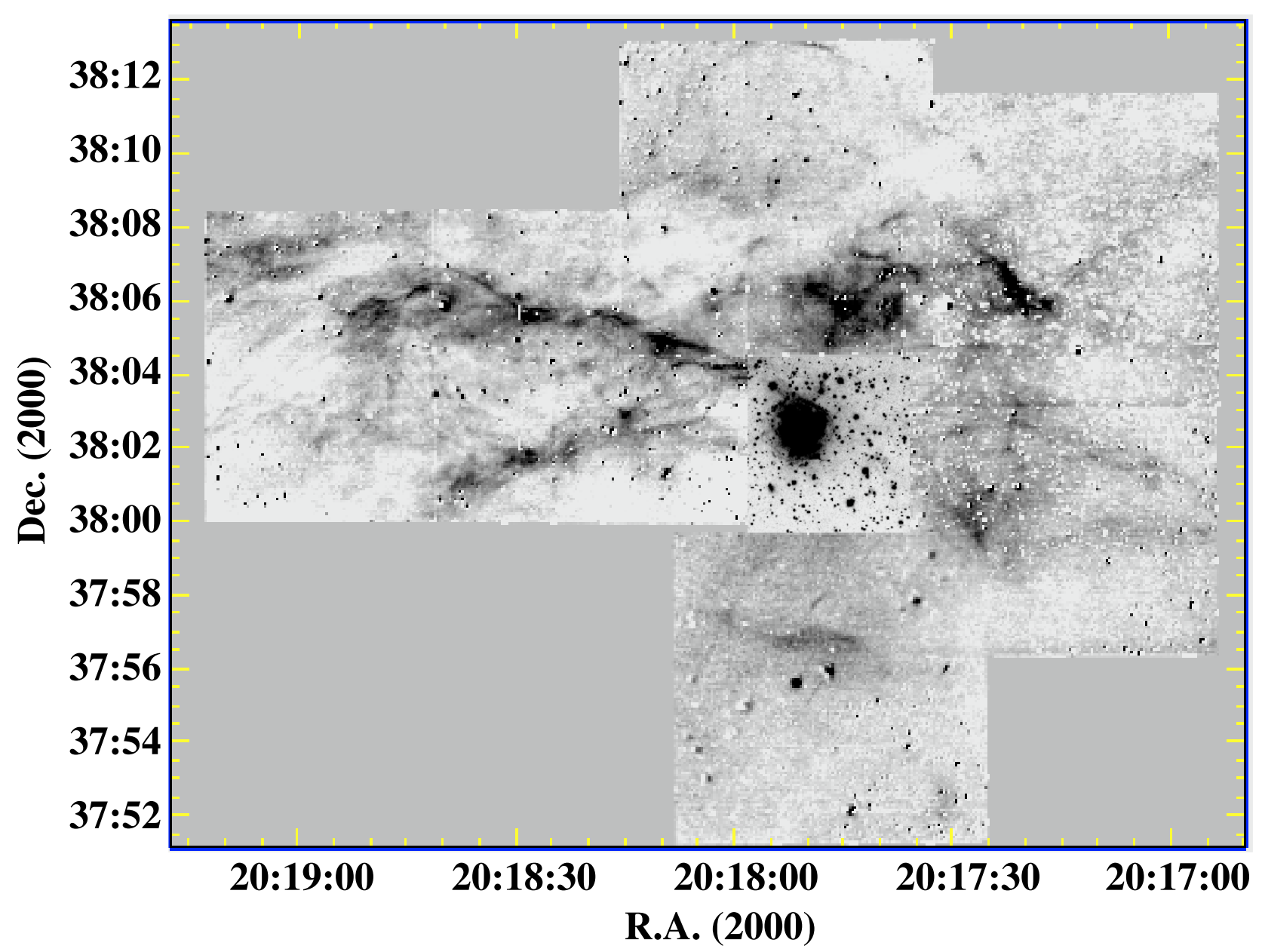

Fig. 1. A mosaic of six [O III]5007 ̊̊ images of the field around P Cygni is shown. These have been continuum subtracted and only residuals from this process, for the brighter stars, are apparent. The central panel is filled by part of the original [N II]6584 ̊̊image from O'Connor et al. (1998) where an occulting mask suppressed the image of the 4.8 mag star P Cygni. This is presented to show the extent of the outer shell, OS and the connections to the various GLs.

respectively. That in Fig. 1 displays the brighter filaments to advantage. The continuum image has been subtracted to suppress the rich star field, whereas in Fig. 2 fainter emission becomes more apparent. The latter is not continuum subtracted. The central panel of Fig. 1 containing P Cygni itself has been filled in with a section of the original (O'Connor et al. 1998) [N II]6584 $\AA$ image where an occulting mask suppressed the bright central star, consequently the eastern, and newly detected northern (see below) GLs can be seen to be apparantly projecting from the OS around P Cygni. The 1.6 arcmin diameter OS which surrounds P Cygni is the dark feature in this central panel.

All of the [O III] $5007 \AA$ emission features shown as contours in Fig. 1 of Meaburn et al. (2004) are confirmed as real here in Figs. 1 and 2. In particular, the southern and eastern counterparts to the original GL are prominent. However, because of both the higher sensitivity and resolution, and the absence of artifacts many new and relevant features are revealed. In Fig. 1a bright [O III] $5007 \AA$ emitting ridge can be seen projecting from just to the north of P Cygni. The [N II]6584 $\AA$ inset image in Fig. 1 shows that this northern ridge continues south to the edge of the OS and points directly back to P Cygni. Fainter and more extensive "lobes" $\approx 7$ ' across can now be seen to the north, east and south of the star. Incidentally, wide absorption lanes of foreground dust can be also seen in Fig. 1 which cause patchy extinction of the [O III] $5007 \AA$ line over this field with the effect that in some places only parts of the extensive filamentary features are observed. Some degree of "bipolar" symmetry exists between pairs of the faint extensive lobes. Moreover, the darker greyscale representation of the same image (now with stars present) in Fig. 2 emphasises how this general [O III]5007 $\AA$ emission is concentrated on P Cygni unlike any other part of the general field in this emission line.

\section{Discussion}

The wider discussion involving both the morphology and kinematics of the GL nebulosity around both P Cygni and related LBV stars in Meaburn et al. (2004) will not be repeated here. Most of the same conclusions stand. The purpose of the present paper is to report new observations, that help to confirm the direct relationship between the surrounding, extensive, highexcitation filamentary GL nebulosity and P Cygni. This association was always left conservatively in doubt after the previous more limited observations.

Here (Figs. 1 and 2), P Cygni appears to be completely surrounded by overlapping, elongated, shell-like filamentary structures over NS and EW diameters of $16^{\prime}(8.4 \mathrm{pc})$ and $20^{\prime}$ (10 pc), respectively, where the distance to this star is $1.8 \mathrm{kpc}$ (van Schewick 1968; Lamers et al. 1983). The [N II]6584 $\AA$ and $\mathrm{H} \alpha$ line profiles (Meaburn et al. 1999, 2000, 2004) over these 


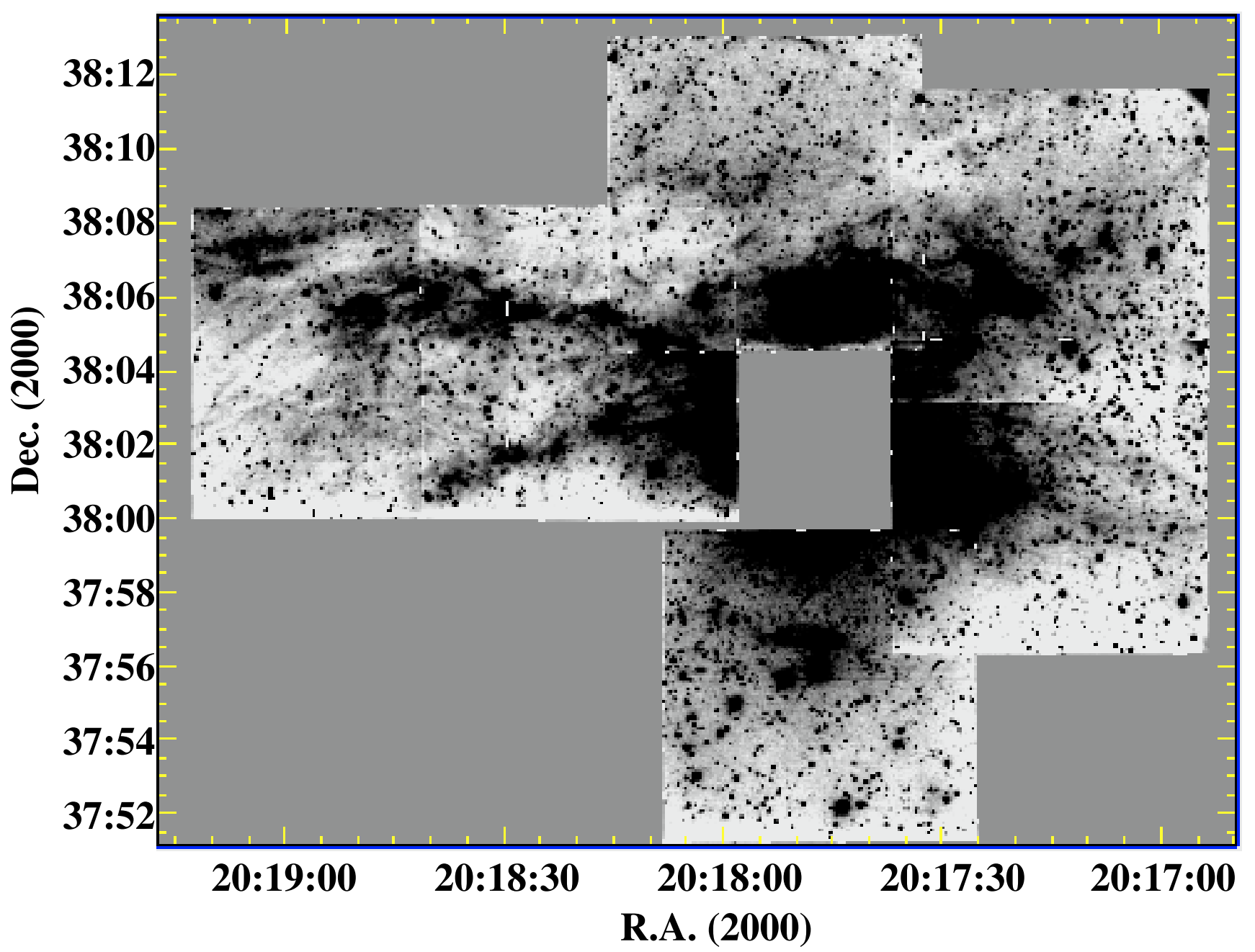

Fig. 2. A darker presentation of the same mosaic of [O III] $5007 \AA$ that is shown in Fig. 1 is presented. The continuum light of stars has not been subtracted and the central panel is no longer filled by the previous image shown in Fig. 1. The concentration of [O III] $5007 \AA$ emission around P Cygni is emphasised.

GLs have many of the velocity characteristics of those of the optical filaments of evolved supernova remnants and, in the limited high spectral resolution observations so far obtained, exhibit predominantly receding radial velocities with respect to $V_{\text {sys }}$ though coming to this value close to the star. However, it is this distribution of lobes and shells, apparently centered on P Cygni that is compelling evidence for a direct association. The positive radial velocities found in the previous limited kinematical observations are still explicable (Meaburn et al. 2004) as a "breeze" as P Cygni moves towards the observer at high velocity with respect to it local ambient medium.

The images in Figs. 1 and 2 reveal the multiple lobes and shells, each $\approx 5 \mathrm{pc}$ across, that must be a consequence of continuous eruptions of P Cygni over a period of $2400-10^{5} \mathrm{yr}$ ago. The lower limit is the well-determined (Meaburn et al. 2000) dynamical age of the OS (shown in the inset in Fig. 1) and the upper limit is that estimated (Meaburn et al. 2004) for the extremities of the bright eastern GL also prominant in Fig. 1. All of these features then precede the observed 1600 AD "great outburst" of P Cygni (de Groot 1969) but are within the duration of the LBV phase expected for a $50 M_{\odot}$ star (Humphreys \& Davidson 1994 and note that Turner et al. 2001 suggested P Cygni had a progenitor mass as low as $25 M_{\odot}$ ). It is now thought most unlikely that the alternative suggestion, that unrelated
SNR optical filaments along the same sight-line are being observed. It is the symmetry of the [O III]5007 $\AA$ shells and lobes, and of the general [O III]5007 $\AA$ emission, around P Cygni combined with the previous kinematical associations (Meaburn et al. 2004) that eliminates this possibility. Spatially-resolved profiles of the [O III]5007 $\AA$ line are now required over the whole field to investigate the dynamics of these ejected phenomena more thoroughly. So far only receding motions have been detected. Also low-dispersion spectra are required to measure the velocities of the shocks that are undoubtably exciting these extensive [O III]5007 ^ emitting filaments. Incidentally, the rather unstructured and inhomogeneous nature of the nebulosity around P Cygni (compared with planetary nebulae, for example) is reminiscent of other high-mass evolved stars; WR 124 is surrounded by a clumpy ring of ejecta that may have originated in a preceeding LBV phase (Grosdidier et al. 1998; van der Sluys \& Lamers 2003).

Acknowledgements. We acknowledge the help of J. Alikakos and S. Akras as well as the support of the staff at Skinakas observatory during these observations. Skinakas Observatory is a collaborative project of the University of Crete, the Foundation for Research and Technology-Hellas, and the Max-Planck-Institut für extraterrestrische Physik, Garching. M.P.R. is supported by the IRCSET, Ireland. 


\section{References}

Barlow, M. J., Drew, J. E., Meaburn, J., \& Massey, R. M. 1994, MNRAS, 268, L29

Boumis, P., Mavromatakis, F., Paleologou, E. V., \& Becker, W. 2002, A\&A, 396, 225

Conti, P. S. 1984, in Observational Test of Stellar Evolution Theory, ed. A. Maeder, \& A. Rensini (Dordrecht: Reidel), 233

Davidson, K., Moffat, A. F. J., \& Lamers, H. J. G. L. M. 1989, Physics of Luminous Blue Variables (Dordrecht: Kluwer)

de Groot, M. 1969, Bull. Astron. Inst. Neth., 20, 225

Grosdidier, Y., Moffat, A. F. J., Joncas, G., \& Acker, A. 1998, ApJ, 506, L127

Humphreys, R. M. 1989, in Physics of Luminous Blue Variables, ed. K. Davidson, A. F. J. Moffat, \& H. J. G. L. M. Lamers (Dordrecht: Kluwer), 3 Humphreys, R. M., \& Davidson, K. 1994, PASP, 106, 1025
Johnson, D. R. H., Barlow, M. J., Drew, J. E., \& Brinks, E. 1992, MNRAS, 255, 261

Lamers, H. J. G. L. M., de Groot, M., \& Cassatella, A. 1983, A\&A, 128, 299 Lasker, B. M., Russel, J. N., \& Jenkner, H. 1999, in the HST Guide Star Catalog, version 1.1-ACT, The Association of Universities for Research in Astronomy, Inc.

Meaburn, J., López, J. A., Barlow, M. J., \& Drew, J. E. 1996, MNRAS, 283, L69 Meaburn, J., López, J. A., \& O’Connor, J. A. 1999, ApJ, 516, L29

Meaburn, J., O’Connor, J. A., López, J. A., et al. 2000, MNRAS, 318, 516

Meaburn, J., Boumis, P., Redman, M. P., López, J. A. \& Mavromatakis, F. 2004, A\&A, 422, 603

O’Connor, J. A., Meaburn, J., \& Bryce, M. 1998, MNRAS, 300, 411

Turner, D. G., Welch, G., Graham, M., et al. 2001, JAVSO, 29, 73

van Schewick, H. 1968, Z. Astrophys., 68, 229

van der Sluys, M. V., \& Lamers, H. J. G. L. M. 2003, A\&A, 398, 181 